\title{
PERCEPÇÃO DA ENFERMAGEM SOBRE AVALIAÇÃO DA DOR ONCOLÓGICA
}

Júlio César Coelho do Nascimento ${ }^{*}$, Jessica da Silva Campos ${ }^{1}$, Virgínia de Paula Vieira ${ }^{2}$ \& Mayro Celso Ramos Barbosa ${ }^{3}$

NASCIMENTO, J.C.C.; CAMPOS, J.S.; VIEIRA, V.P.; BARBOSA, M.C.R. Percepção da enfermagem sobre avaliação da dor oncológica. Perspectivas Online: Biológicas \& Saúde, v.10, n.32, p.51-61, 2020.

\section{RESUMO}

A dor é um fenômeno complexo e de difícil mensuração, principalmente na população com câncer em tratamento paliativo. Portanto, a equipe de enfermagem é extremamente importante nesse processo. Assim, o presente estudo objetivou analisar a percepção da equipe de enfermagem frente à avaliação da dor em pacientes com câncer em cuidados paliativos. Tratou-se de um estudo descritivo de abordagem qualitativa, realizado com 10 profissionais de enfermagem (três enfermeiros e sete técnicos de enfermagem) de um hospital de grande porte referência em oncologia localizado na cidade de Goiânia-GO. A coleta de dados ocorreu em novembro de
2017 no setor de cuidados paliativos, por meio de um questionário semiestruturado contendo perguntas abertas e fechadas. Dentre os pesquisados, a idade média predominante foi de 44,5 anos. Com predominância o sexo feminino $(80 \%)$ todos os profissionais com mais de 4 anos de experiências no setor. Os dados foram analisados e os resultados apontaram que a avaliação da dor ainda continua sendo um processo negligenciado, sobretudo no tratamento paliativo. Com base nesses resultados, torna-se necessário uma capacitação dos profissionais de enfermagem no que se refere à importância da avaliação e, sobretudo no manejo da dor.

Palavras-chave: Câncer; Enfermagem; Dor; Cuidados Paliativos. 


\begin{abstract}
The pain is a complex phenomenon and difficult to measure, especially in the population with cancer in palliative treatment. Therefore, the nursing team is extremely important in this process. Thus, the present study aimed to analyze the perception of the nursing team regarding the evaluation of pain in patients with cancer in palliative care. This was a descriptive-exploratory cross-sectional study carried out with 10 nursing professionals (3 nurses and 7 nursing technicians) of a large referral hospital in oncology located in the city of GoiâniaGO. Data collection took place in

November 2017 in the palliative care sector, through a semi-structured questionnaire containing open and closed questions. Among those surveyed, the prevailing mean age was 44.5 years. With a predominance of women $(80 \%)$ all professionals with more than 4 years of experience in the sector. The data were analyzed and the results indicated that the evaluation of pain still remains a neglected process, especially in the palliative treatment. Based on these results, it is necessary to train nurses in the importance of evaluation and, especially, in pain management.
\end{abstract}

Keywords: Neoplasms; Nursing; Pain; Palliative care.

\footnotetext{
${ }^{1}$ Enfermeiros, Mestrandos pelo Programa de Pós-graduação em Assistência e Avaliação em Saúde, Faculdade de Farmácia, Universidade Federal de Goiás, R. 240, S/n - Setor Leste Universitário, Goiânia - GO, CEP: 74605-170.

${ }^{2}$ Acadêmica de Enfermagem - Faculdade Noroeste. Av. Mangalô, no 2385 - St. Morada do Sol, Goiânia - GO, CEP: 74475115 .

${ }^{3}$ Estudante de Enfermagem - Nível Técnico. Colégio Oswaldo Cruz R. 20, 796 - St. Central, Goiânia - GO, CEP: 74020-170. (*) e-mail: prof.julioccnascimento@gmail.com

Data de recebimento: 14/11/2019. Aceito para publicação: 21/02/2020.
} 


\section{INTRODUÇÃO}

Os profissionais de saúde se deparam com situações desafiantes durante o processo de enfrentamento do câncer. Dentre elas, salienta-se a dor por ser um sinal e sintoma altamente apresentado e relatado. A Associação Internacional para o Estudo da Dor (IASP) define a dor como uma experiência desagradável, relacionada ao sistema sensorial e emocional descrita em termos de lesões teciduais (GOMES, 2007; WATERKEMPER; REIBNITZ, 2010).

O conceito de dor abrange os aspectos somáticos causados pela dor que Cicely Saunders (1993) conceituou como dor total. Saunders foi uma das fundadoras do St. Christopher's Hospice na Inglaterra. Foi ela que conceituou Dor Total a partir dos elementos emocionais, psicológicos, sociais e espirituais (ANDRADE; TORRES, 2015; NAIME, 2013).

A síndrome da dor total referido por Saunders (1993) é a expressão mais forte do sofrimento humano que pode ser experimentada por cerca de $75 \%$ dos pacientes oncológicos em Cuidados Paliativos (CP) (MUÑOZ; MONJE, 2010).

De acordo com Silva et al., (2011) geralmente na fase inicial, o câncer é indolor. No entanto, a manifestação clínica pode ocorrer em 33\% dos pacientes em tratamento precoce. No estágio avançado, $90 \%$ dos pacientes relatam dor de moderada a severa, portanto, a dor total só é alcançada nesse estágio.

A dor pode estar relacionada à invasão direta do tumor (local e sistêmica), o próprio tratamento do câncer (cirurgia, quimioterapia ou radioterapia, e biópsia) ou problemas não relacionados ao câncer (por exemplo: hérnia de disco e neuropatia diabética). Fatores psicossociais (depressão, ansiedade, catastrofização e cognição) podem influenciar a percepção da dor e contribuir para a intensidade da dor total (MINSON et al., 2012).

A dor relacionada ao câncer propicia-se mais de um tipo: incidental (transitória, súbita causada por algum movimento, tosse, evacuação), intermitente (dor episódica), neuropática (decorrente de lesão total ou parcial), nociceptiva ou mista (decorrente de estimulação química ou física, que pode ser constante ou intermitente) (MINSON et al., 2012).

Alonso (2013) afirma que o alívio da dor é uma questão de direitos humanos juntamente com os cuidados paliativos. Portanto, o controle da dor necessita de atenção multiprofissional, para garantir o direito de pacientes com câncer uma qualidade de vida.

Em 1996, segundo a Sociedade Brasileira para o Estudo da dor (SBED), o presidente da Sociedade Americana de Dor, James Campbell citou e reconheceu a dor como quinto sinal vital e afirmou ainda que "se a dor fosse aliviada com o mesmo zelo como os outros sinais haveria uma melhor chance de promover tratamento adequado" (AMARAL; CALASANS, 2013, p. 151). Contudo, de acordo com Carvalho, Nóbrega e Garcia (2013), só em janeiro do ano 2000, a dor foi realmente considerada como quinto sinal vital, o que evidencia a negligência.

Corroborando com Campbell, Bottega e Fontana (2010), acreditam que a dor descrita como o quinto sinal vital, deve ser avaliada automaticamente bem como a temperatura corporal, pulso/frequência cardíaca, pressão arterial e frequência respiratória. Deve ser avaliada por meio de instrumentos que possam transcrever sua intensidade. Ribeiro, Barreto e Sousa (2011) sugerem para avaliação da dor, adoção de um protocolo em instituições de saúde que deve ser seguido por todos envolvidos no atendimento aos pacientes. 
Para Pinto, Santoro e Silva (2012) a avaliação é de grande relevância na compreensão da dor, na identificação de sua origem e consequentemente na análise do seu impacto sobre o indivíduo considerando principalmente aspectos de vida do paciente, assim como suas experiências sensoriais, afetivas, comportamentais e cognitivas.

Dentre esses instrumentos disponíveis para avaliar a intensidade da dor, Sorin, José e Sarria (2012), destacam: a Escala Numérica, que é mais utilizada, a Escala Analógica Visual, e as Escalas de Faces. Essas escalas são chamadas de unidimensionais, servem para avaliar somente a intensidade da dor (NAIME, 2013).

Além das escalas referidas, Naime (2013) relata que existem as escalas multidimensionais, usadas para uma avaliação mais complexa. Dentre elas destacam Edmonton SymptomAssessment System (ESAS) e McGill Questionnarie.

As escalas unidimensionais servem para medir a intensidade da dor e as escalas multidimensionais aferem e avaliam o efeito da dor diariamente e na qualidade de vida. Para a categoria das Escalas unidimensionais, as mais utilizadas são a escala visual numérica, que é instrumento simples de linha reta com enumeração de 0 a 10 , sendo o início dessa linha 0 , representando "sem dor" e 10 com apresentação da dor máxima (AGUIAR; PINHEIRO, 2019).

Ainda dentro dessa categoria, temos a escala visual analógica (EVA), escala de avaliação verbal e escala de expressões faciais. A EVA consiste na avaliação dos componentes sensitivos da dor em uma linha de $10 \mathrm{~cm}$, (sem dor em uma extremidade, até dor máxima na outra extremidade), já a escala de avaliação verbal é feita sem auxílio de instrumento, nesse tipo de escala o paciente quantifica a experiência dolorosa usando frases que representam diferentes intensidades da dor (nenhuma dor, dor leve, dor moderada, dor intensa) (AMARAL; CALASANS, 2013).

A escala de faces criada por Wong Baker também é conhecida por Wong Baker Face Scale. Conforme caracteriza Morete e Minson (2010), a primeira figura é muito sorridente e as expressões vão se transformando, mostrando graus crescentes de tristeza, até chegar a última que é muito triste. Diante da escala, o paciente escolhe a face que julga mais parecida com a sua.

Quanto as escalas multidimensionais a literatura destaca o Inventário Breve da Dor (IBD) e o McGill de Dor (McGill Questionnaire - MPQ). O IBD é um instrumento eficaz para avaliação da dor, e esta tem sido bastante usada em pacientes com câncer. Inclui um esquema para anotar a localização da dor, perguntas a respeito da intensidade atual, média, e a pior, usando a escala de avaliação de 0 a 10 (MORETE; MISON, 2010).O McGill de Dor é um questionário que avalia a qualidade da dor, desenvolvido pelo Dr. por Melzack na Universidade McGill, em Montreal no Canadá no ano de 1975 (NAIME, 2013). Esse questionário avalia aspectos da dor por meio de descritores que o paciente escolhe para expressar a sua dor. Divididos em quatro grupos, os descritores são: sensorial discriminativo, afetivo motivacional, avaliativo cognitivo e miscelânea (SANTOS, et al., 2006).

Martinez, Grassi e Marques (2011) explicam que os descritores podem ter valor máximo de 20. Já o índice de dor é calculado pela somatória dos valores de intensidade de cada descritor (0-5), tendo esse o máximo de 78. MPQ conta com um diagrama corporal para melhor localização da dor e avaliação da dor quanto a sua periodicidade e duração. 
Outro instrumento, também desenvolvido no Canadá, foi a Escala de Edmonton (ESAS). Criada em 1991, no Hospital Geral de Edmonton. Em 2010, foi revisada no Brasil com o objetivo de facilitar o entendimento dos pacientes. Esse instrumento auxilia na detecção e monitoramento de sintomas em pacientes com câncer em CP avaliando a combinação de sintomas físicos e psicológicos (MONTEIRO; ALMEIDA; KRUSE, 2013).

A dor, assim como os outros sinais vitais representa um indicador muito importante que pode evidenciar possíveis complicações do estado de saúde apresentado pelo paciente, portanto se torna necessário a abordagem da mesma como quinto sinal vital (MORAIS et al., 2009).

Em um estudo realizado por Alonso (2013), na cidade de Buenos Aires afirmou que o controle da dor é o principal motivo para os pacientes buscarem os serviços de CP. Nesse estudo, os pacientes relataram negligência no manejo da dor nos centros não especializados.

No Hospital da Universidade de Toronto - Canadá, Sunnybrook Odette Cancer Centre, foi realizado um estudo que avaliou a prevalência de dor de 1.000 pacientes diante do programa de CP prestados pela instituição. Dentre esses, 25.4\% (254) relataram dor leve, 19,6\% (196) relataram dor moderada e 46,5\% (465) relataram a dor grave (MITERA et al., 2010).

A dor é o principal fator responsável pela diminuição da qualidade de vida dos pacientes oncológicos. A partir desse reconhecimento, a importância da avaliação da dor adequada resultou no desenvolvimento de vários instrumentos para a avaliação da dor e, consequentemente, em um tratamento adequado (KWON et al., 2013).

Como a dor é um sintoma predominante em pacientes oncológicos, principalmente naqueles que estão em cuidados paliativos, tornou-se uma questão amplamente discutida pelos profissionais de saúde. No entanto, percebe-se que a avaliação da dor é um processo subestimado, devido à subjetividade dos instrumentos disponíveis para uma avaliação precisa. Levando em consideração que a equipe de enfermagem passa mais tempo com o paciente, questiona-se sobre o conhecimento da equipe quanto à importância da avaliação da dor.

Geralmente a dor no câncer pode ser tratada, no entanto, esse processo exige uma abordagem multidisciplinar que contenha conhecimento da fisiopatologia da dor, bem como a farmacologia dos analgésicos e o manejo das questões psicossociais (MINSON et al., 2012). O alívio da dor e a promoção de conforto são intervenções essenciais que envolvem além de conhecimento científico e habilidade técnica, questões humanitárias e éticas da prática da enfermagem. A importância do estudo da dor deve-se ao fato de que a sensação gera estresse, sofrimento e desconforto para o paciente e sua família.

Nessa perspectiva, o presente estudo investigou a percepção da equipe de enfermagem de um hospital de grande porte localizado na cidade de Goiânia-GO frente à avaliação da dor em pacientes com câncer em cuidados paliativos. Acreditamos que esta investigação seja imprescindível para a promoção do cuidado integral e humanizado.

\section{METODOLOGIA}

Trata-se de estudo do tipo descritivo de abordagem qualitativa. Esse método propiciou a 
caracterização e a mensuração de variáveis, fornecendo informações sobre o fenômeno estudado. A abordagem qualitativa permitiu compreender o significado das relações humanas, permeadas pelas emoções e sentimentos vivenciados no dia a dia, por meio da percepção, intuição e subjetividade (MARCONI; LAKATOS, 2005).

Antes do procedimento de investigação, o trabalho foi submetido e aprovado pelo Comitê de Ética em Pesquisa do Hospital Araújo Jorge - ACCG, sob parecer de nº 2.385.221.

O Hospital Araújo Jorge é referência em oncologia e está localizado na cidade de Goiânia-GO, região Centro-Oeste do Brasil. Trata-se de uma instituição de saúde privada e filantrópica. Esse hospital é considerado referência no tratamento de todos os tipos de câncer no Centro-Oeste, atendendo também pacientes de outras regiões brasileiras, notadamente Norte e Nordeste. Há mais de 50 anos o Hospital Araújo Jorge está no grupo dos maiores e melhores hospitais brasileiros especializados em cancerologia, atendendo por mês cerca de 28.000 pacientes de todas as idades.

O hospital conta com o Grupo de Apoio Paliativo ao Paciente Oncológico (GAPPO) que desenvolve um trabalho interdisciplinar que está de acordo com os princípios dos cuidados paliativos, que visam: promover o alívio da dor e de outros sintomas estressantes, reafirmar a vida e possibilitar que a morte seja vista como um processo natural, integrar aspectos psicossociais e espirituais ao cuidado, oferecer auxilio para o paciente viver tão ativamente quanto possível, oferecer suporte que auxilie a família e aos entes queridos a sentirem-se amparados durante todo o processo da doença.

A equipe de enfermagem da referida unidade é composta por um coordenador, quatro supervisores, três enfermeiros assistencialistas, dezesseis técnicos de enfermagem e seis auxiliares de enfermagem. O setor possui vinte e nove leitos sendo seis particulares e vinte e três do Sistema Único de Saúde (SUS).

Foram convidados a participar da pesquisa, atuantes no setor de cuidados paliativos em oncologia desse hospital, que preencheram os seguintes critérios: atuar como enfermeiro, técnico ou auxiliar de enfermagem; estar no setor oncológico por no mínimo seis meses (tempo mínimo para que o profissional possa se familiarizar com o ambiente, rotina e pessoas envolvidas com o trabalho do setor) e não estar no período de férias ou licença médica.

A coleta de dados ocorreu no mês de novembro de 2017, com convite aos enfermeiros, técnicos e auxiliares de enfermagem. Nele constou uma breve apresentação do tema, com escrita simples e de forma clara, sendo especificado o local, data, horário e identificação do pesquisador responsável. Após a apresentação, os participantes tiveram um espaço aberto para o esclarecimento de dúvidas que foram respondidas pelo pesquisador. Finalizada essa etapa, três enfermeiros e sete técnicos de enfermagem aceitaram participar da pesquisa.

Posteriormente os participantes foram reunidos com a presença do pesquisador, de forma reservada, para a entrega individual do Termo de Consentimento Livre e Esclarecido, o qual foi lido com o acompanhamento dos presentes, para que fosse compreendido, sendo sanadas possíveis dúvidas antes da sua assinatura.

$\mathrm{Na}$ etapa seguinte foi entregue a cada profissional um questionário semiestruturado elaborado pelos autores, composto por cinco perguntas iniciais objetivando caracterizar os participantes do estudo e seu conhecimento acerca da avaliação da dor nos cuidados no final de vida. O questionário foi lido pelo pesquisador responsável com o acompanhamento dos 
presentes e explicado seus itens, um a um, sendo sanadas quaisquer dúvidas. Após a compreensão do questionário pelos profissionais, os mesmos foram solicitados a respondê-lo individualmente e ao término foi recolhido pelo pesquisador de forma a garantir o sigilo das informações.

\section{RESULTADOS E DISCUSSÃO}

Nesse estudo, descreveu-se a percepção dos profissionais de enfermagem a cerca da avaliação da dor no tratamento paliativo em um hospital referência em oncologia localizada na cidade de Goiânia.

De acordo com a formação profissional, faixa etária e o gênero dos profissionais de enfermagem, a maior parte da amostra, sete (70\%) foi composta por profissionais técnicos em enfermagem, com idade média de 44,5 e o gênero predominante foi o feminino, oito (80\%).

Tabela 1. Caracterização dos participantes do estudo segundo faixa etária, sexo e formação profissional. Goiânia, Goiás, Brasil, 2018.

\section{CARACTERIZAÇÃO}

FAIXA ETÁRIA

Até 30 anos

Entre 31 e 40 anos

Entre 41 e 50 anos

Entre 51 e 60 anos
FORMAÇÃO PROFISSIONAL

Técnicos de enfermagem

Enfermeiros

TOTAL

\begin{tabular}{llll}
\multicolumn{2}{c}{ SEXO MASCULINO } & \multicolumn{2}{c}{ SEXO FEMININO } \\
$\mathrm{n}$ & & $\mathrm{n}$ & $\%$ \\
0 & 0 & 1 & 10 \\
2 & 20 & 1 & 10 \\
0 & 0 & 4 & 40 \\
0 & 0 & 2 & 20 \\
7 & 70 & & \\
3 & 30 & &
\end{tabular}

Fonte: autoria própria

Em relação ao conhecimento sobre a avaliação da dor, verificou-se a insegurança dos participantes em responder ao questionamento, no entanto, constatou-se que apenas quatro (40\%) profissionais sabiam sobre o assunto e seis (60\%) não sabiam sobre o assunto (gráfico 1). Além disso, apenas três (30\%) entrevistados afirmaram avaliar a dor com frequência. 
Gráfico 1 - Conhecimento dos profíssionais sobre a avaliação da dor. Goiânia, Goiás, Brasil, 2018.

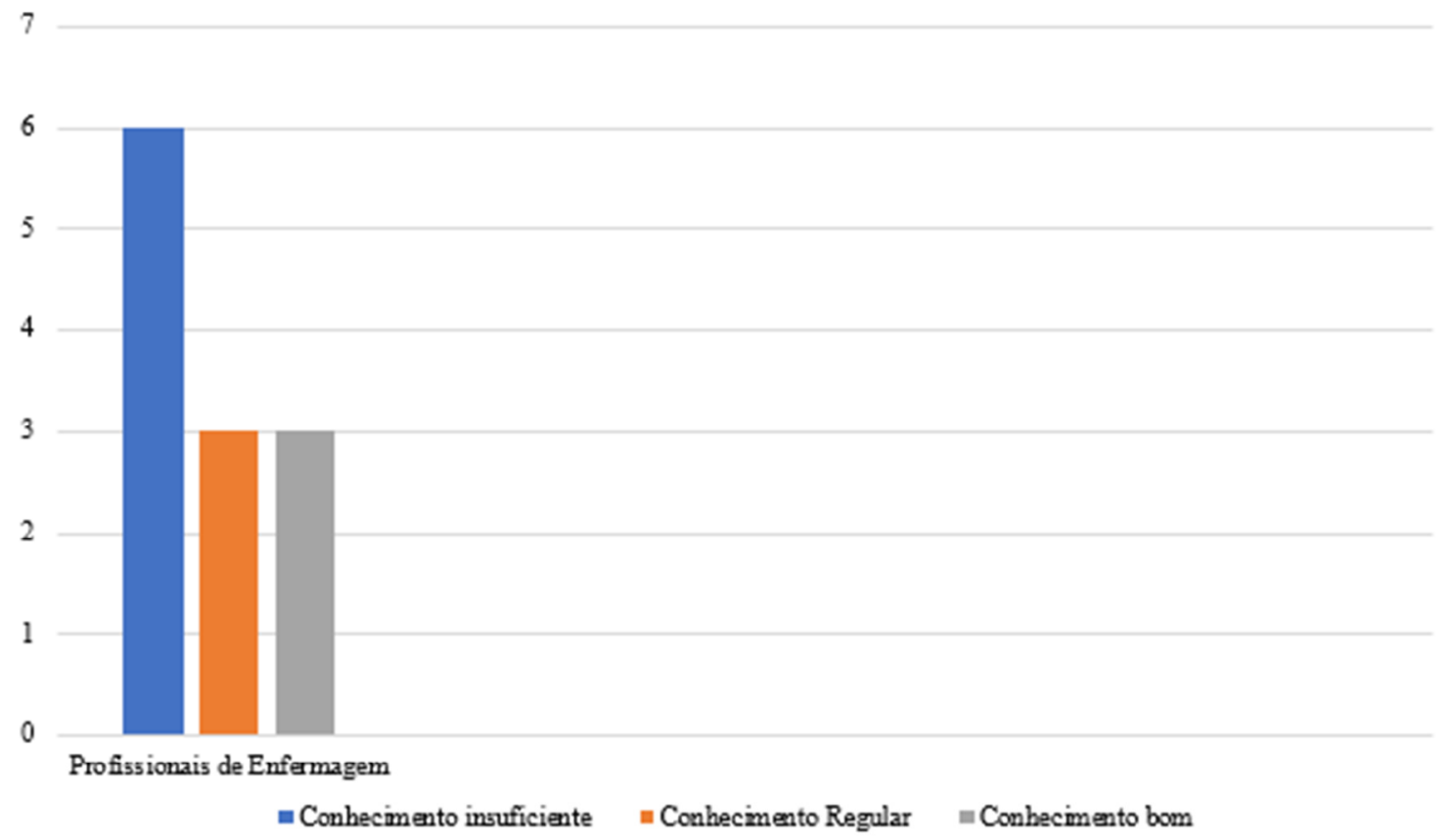

Fonte: Os autores

Observa-se que o número de profissionais com conhecimento considerado suficiente é baixo. Assim como no estudo realizado por Payen et al (2001) na Itália que investigou o conhecimento dos enfermeiros no manejo de dor em pacientes oncológicos, a média de acertos foi superior a $62 \%$, sendo considerada como conhecimento inadequado.

Quanto aos instrumentos para avaliar a dor, quatro (40\%) se ancoram na escala de faces, três (30\%) relatam quanto à adesão da escala verbal e apenas um (10\%) relatou conhecer as escalas para avaliar a dor (escala analógica, escala de faces, escala de cores, entre outras).

Questionados sobre a eficácia do método escolhido para avaliar a dor, apenas dois (20\%) acreditam que um tipo de instrumento específico é capaz de avaliar todos os pacientes, independente de sua condição clínica. Oito (80\%) relataram que o instrumento escolhido para avaliar a dor deve ser compatível com o estado clínico do paciente.

Os resultados mostraram que oito (80\%) dos entrevistados reconhecem a dor como um indicador importante e que não deve ser negligenciado.

A equipe de enfermagem é o grupo que fica o maior tempo em contato com pacientes internados em hospitais. A natureza da profissão, que inclui a prestação de cuidados físicos e a execução de procedimentos diagnósticos e terapêuticos, torna-a um elemento fundamental na avaliação da dor. No entanto, mesmo com a evolução dos serviços de saúde e dos fármacos, nota-se que a dor não é devidamente avaliada em pacientes com câncer em tratamento paliativo. Certamente, por esse motivo, eles não recebem uma terapêutica adequada (SILVA et al., 2011). 
Segundo a literatura, as escalas para avaliar a dor são chamadas de unidimensionais e multidimensionais. Para verificar a intensidade indica-se o uso das unidimensionais (Escala de Faces, Analógica e Escala Numérica), todavia elas são limitadas. Para avaliar o efeito na dor na qualidade de vida usam-se as escalas multidimensionais (Edmonton Symptom Assessment System (ESAS) e McGill Questionnarie).

Contradizendo Sorin, José e Sarria (2012), a Escala Numérica não é a mais utilizada pela equipe de enfermagem do Hospital Araújo Jorge. As escalas mais citadas foram as escala Analógica Visual e Escalas de Faces. Notadamente percebe-se falha na assistência prestada aos pacientes em tratamento paliativo, uma vez que as escalas referidas servem para avaliar somente a intensidade da dor (NAIME, 2013).

As escalas multidimensionais não foram citadas em nenhum momento pelos participantes desta pesquisa, o que evidencia que a avaliação da dor ainda continua sendo um processo negligenciado mesmo no tratamento paliativo que é um processo enfrentado com quadros álgicos de níveis severos.

$\mathrm{Na}$ coleta dos dados, identificamos que apenas dois (20\%) fazem avaliação da dor concomitantemente a aferição dos sinais vitais conforme proposto por Bottega e Fontana (2010).

De tal modo, os resultados demonstraram que os profissionais da instituição pesquisada não exercem a autonomia profissional, visto que foi informado que em muitas situações a avaliação da dor é realizada conforme orientação de outro profissional. Ressalta-se que a autonomia corresponde à realização de ações de enfermagem por meio da utilização de habilidades, conhecimentos e atitudes para tomar decisões e resolver situações no seu espaço de atuação (FENTANES, 2011).

\section{CONCLUSÕES}

Ao término desta pesquisa, alcançamos os objetivos propostos, considerando que este trabalho teve grande importância por mostrar uma realidade no que se refere à avaliação da dor de pacientes com câncer em tratamento paliativo do hospital referência na cidade de Goiânia-GO, que de forma direta ou indiretamente reflete na qualidade da assistência desenvolvida.

Os resultados levam a concluir que os profissionais de enfermagem, em seu maior número, apresentam baixo nível de conhecimento no que se refere à avaliação da dor.

Dentre os pesquisados encontram-se profissionais que possuem mais de quatro anos de experiência lidando com pessoas com dor oncológica. No entanto, observou-se um número significativo de profissionais de enfermagem, que ainda apresenta níveis insatisfatórios de conhecimento, reforçando o que alguns autores vêm mostrando quanto ao conhecimento dos profissionais de enfermagem sobre a dor, quando afirmam ser este insuficiente para o manejo dessa situação, tão usual nos pacientes e em especial nos pacientes oncológicos.

Assim, compreende-se ser necessário um melhor preparo do profissional para o cuidado prestado a pacientes com dor e uma educação continuada. 
Uma das limitações encontradas na realização deste estudo foi a escassez de pesquisas realizadas no Brasil sobre o assunto. Portanto, sugere-se a realização de futuros estudos sobre a temática para conhecer a realidade do manejo da dor em pacientes oncológicos sob cuidados paliativos.

\section{REFERÊNCIAS}

AGUIAR, D.S.; PINHEIRO, I.M. Multidimensional instruments validated in Brazil for pain evaluation in the elderly: narrative review. BrJP. São Paulo, v.2, n.3, p.289-92, 2019.

ALONSO, J.P. El tratamiento del dolor por cáncer en el final de la vida: estudio de caso en um servicio de cuidados paliativos de la Ciudad Autónoma de Buenos Aires. Salud Colectiva, v.9, n. 1, p.41-52, 2013.

AMARAL, J.B.; CALASANS, M.T.A. As dimensões da dor na pessoa sob cuidados paliativos. In: SILVA, R.S.; AMARAL, J.B.; MALAGUTTI, W. Enfermagem em Cuidados Paliativos: cuidando para uma boa morte. São Paulo: Martinari, 2013, p. 149-164.

ANDRADE, D.S.; TORRES, V, P, S. Perspectivas do enfermeiro frente aos cuidados para alívio da dor no paciente terminal oncológico. Rev. Perps. online: Biol \& Saúde, v.19, n.5, p.63-77, 2015.

BOTTEGA, F. H.; FONTANA, R. T. A dor como quinto sinal vital: utilização da escala de avaliação por enfermeiros de um hospital geral. Texto Contexto Enferm., v. 19, n.2, p. 283290, 2010.

CARVALHO, M. W.A.; NÓBREGA, M.M.L.; GARCIA, T.R. Processo e resultados do desenvolvimento de um Catálogo CIPE $^{\circledR}$ para dor oncológica. Rev. Esc. Enferm. USP, v. 47 , n. 5, p. 1061-1068, 2013.

FENTANES, L.R.C.; HERMANN, A.P.; CHAMMA, R.C. et al. Autonomia profissional do enfermeiro: revisão integrativa. Ver. Cogitare Enferm., v. 16, n.3, p. 530- 535, 2011.

GOMES, P. C. A bioética e a dor: algumas reflexões. In: LEÃO, E. R.; CHAVES, L. D. (Org.). Dor $\mathbf{5}^{\mathbf{0}}$ sinal vital: reflexões e intervenções de enfermagem. São Paulo: Martinari, 2007

KWON, J.H.; HUI, D.; CHISHOLM, L. et al. Experience of Barriers to Pain Management in Patients Receiving Outpatient Palliative Care. Journal of Palliative Medicine, v.16, n.8, p. 908-914, 2013.

MARCONI, M.A.; LAKATOS, E.M. Fundamentos de metodologia científica. $6^{a}$ ed. São Paulo, SP: Atlas, 2005.

MARTINEZ, J.E; GRASSI, D.C.; MARQUES, L.G. Análise da aplicabilidade de três instrumentos de avaliação de dor em distintas unidades de atendimento: ambulatório, enfermaria e urgência. Rev. Bras. Reumato, v.51, n.4, p.299-308, 2011.

MINSON et al. Procedimentos intervencionistas para o manejo da dor no câncer. Einstein, v.10, n. 3, p. 292-295, 2012.

MITERA et al. Retrospective Assessment of Cancer Pain Management in an Outpatient 
Palliative Radiotherapy Clinic Using the Pain Management Index. Journal of Pain and Symptom Management, Toronto - CAN, v.39, n.2, 2010.

MONTEIRO, D.R.; ALMEIDA, M.A; KRUSE, M.H.L. Tradução e adaptação transcultural do instrumento Edmonton Symptom Assessment System para uso em cuidados paliativos. Ver. Gaúcha Enferm., Porto Alegre, v. 34, n.2, p. 163-171.

MORAIS, F. F. et al. Avaliação da dor como quinto sinal vital na classificação de risco: um estudo; com enfermeiros. Ver. Ciênc. \& Saúde, v. 2, n. 2, p. 73-77, 2009.

MORETE, M.C.; MINSON, F.P. Instrumentos para a avaliação da dor em pacientes oncológicos. Ver. Dor, São Paulo,v. 11, n. 2, p. 74-80, 2010.

MUÑOZ, E.; MONJE, D. Valoración de Dolor Total en el Policlínico Alivio del Dolor y Cuidados Paliativos del Complejo Hospitalario San José. Ver. El Dolor, v.19, n. 54, p. 26-34, 2010 .

NAIME, F. F. Manual do tratamento da dor: Dor aguda e dor de origem oncológica: tratamento não invasivo. Barueri, SP: Manole, 2013. p. 18 -19.

PAYEN, J.F.; BRU, O.; BOSSON, J.L. et . Assessing pain in critically ill sedated patients by using a behavioral pain scale. Crit. Care. Med. v.29, n.12, p. 2258-2263, 2001.

PINTO, C.M.I.; SANTORO, D.C.; SILVA, J. Um estudo sobre atividades relacionadas a intervenções de enfermagem controle da dor no cenário da terapia intensiva Rev. Perps. online: Biol. \& Saúde, v.06, n.02, p.70-75, 2012.

RIBEIRO, N. C.; BARRETO, S.C.C.; HORA, E.C. et al. O enfermeiro no cuidado à vítima de trauma com dor: o quinto sinal vital. Rev. Esc. Enferm. USP, São Paulo v. 45, n. 1, p. 146$152,2011$.

SANTOS, C.C.; PEREIRA, L.S.M.; RESEND, M.A. et al. Aplicação da versão brasileira do questionário de dor Mcgill em idosos com dor crônica. Rev. Acta Fisiatr., v.13, n.2, p.75-82, 2006.

SAUNDERS C, SYKES N. The management of terminal malignant disease. 3 ed, Londres:Edward Arnold, 1993.

SILVA, T. O. N.; SILVA, V. R.; MARTINEZ, M.R.; GRADIM, C.V.C. Avaliação da Dor em pacientes oncológicos. Rev. Enferm. UERJ, v. 19, n.3, p. 359-363, 2011.

SORIN, B.M.D.; JOSÉ, E.; SARRIA, M.D. The Management of Pain Metastatic Bone Disease. Rev Cancer Control. v. 19, nº 2, p. 154-166, 2012.

WATERKEMPER, R.; REIBNITZ, K. S. Cuidados Paliativos: a avaliação da dor na percepção de enfermeiras. Ver. Gaúcha Enferm., v. 31, n. 1, p. 84-91, 2010. 